temperatures. There is the analogous effect in liquid helium which becomes superfluid at $2.17 \mathrm{~K}$ and for which we still do not have a completely satisfactory explanation. And, still colder, we have the remarkable series of changes which occurs in the light isotope of helium - liquid helium three.

This is a book which whets the appetite. The treatment is simple and yet the explanations are not so oversimplified that they would worry a scientist. Occasionally the broadcasting voice of the author comes through a little too strongly so that the excitation and marvelling at new phenomena or techniques, which is very necessary via the microphone, is perhaps a little bit too enthusiastic in print. But this is only a minor point. David Wilson has done a good job and it is evident that he enjoyed doing it.

H. M. Rosenberg is University Reader in Physics at the Clarendon Laboratory and Fellow of St Catherine's College, University of Oxford, UK.

\section{Molecular optical activity}

\section{S.F. Mason}

The Molecular Basis of Optical Activity: Optical Rotatory Dispersion and Circular Dichroism. By E. Charney. Pp.376. (Wiley: New York and Chichester, UK, 1979.) $£ 18.30, \$ 39.90$.

For Louis Pasteur, the nonsuperposability of mirror-image molecular structures was the primary characteristic of natural products, as opposed to synthetic substances, and the distinctive physical property of handed molecules, their optical activity, provided him with a demarcation criterion between chemistry and life. The investigation of the optical rotatory power of chiral molecules, fundamentally, their differential interaction with left- and rightcircularly polarized light, played a central role in the development of stereochemistry during the nineteenth and early twentieth centuries. From the 1950 s, however, the chiroptical methods became increasingly overshadowed by more sophisticated physical techniques in structural chemistry, and their particular value in problems concerned with chiral recognition and discrimination was sometimes overlooked. The thalidomide tragedy, for example, could have been averted if this synthetic racemate had been separated into its optical isomers, for only the left-handed (S)-(-)-isomer has teratogenic properties. While occupying a subsidiary place in mainstream chemistry from the 1950 s, the study of the electronic and structural basis of optical activity has proliferated at the periphery, in biophysics, biochemistry and chemical physics. The present book was written from the Laboratory of Chemical Physics, NIH, Bethesda, and the majority of the current theories of optical activity reviewed were first published in the Journal of Chemical Physics, rather than the orthodox physical chemistry journals.

The author's primary aim, which has been largely achieved, is the systematic development of the fundamental principles underlying the modern theory of the optical properties of chiral molecular structures, and of the derived semiempirical models employed in the stereochemical and other applications of chiroptical spectroscopy. After an introductory historical survey, the classical and the quantum theory of the interaction of an assembly of molecules with electromagentic radiation is discussed, with the extension beyond the standard treatment required to account for optical activity, namely, the explicit consideration of terms dependent upon the ratio of the molecular dimensions to the wavelength of the radiation. The particular approximate models covered are those of the "symmetric chromophore', or light-absorbing group, bonded to a dissymmetric array of substituent groups in a chiral molecule, the 'coupled chromophore pair' of a chiral dimer, and the general but more miscellaneous case of the "inherently dissymmetric chromophore'. A grouptheoretical treatment of chirality follows, with applications to the sector rules connecting the stereochemical configuration of an enantiomer with its rotary strength in a given electronic transition. There is a minor confusion in this section. The second of the three classes of point group symmetries listed (p.152) should be partitioned between the first and the third, since there are but two classes, chiral molecules with pure rotation symmetry and achiral structures with rotation-reflection symmetry, which implies the superposability of the mirror-image forms. Subsequent chapters are devoted to individual chromophore systems, the optical activity of polymers and of orientated molecule assemblies, and to recent developments. The latter include luminescence- and fluorescence-detected optical activity, and, more particularly, vibrational optical activity in both infrared absorption and Raman scattering spectroscopy.

The book is well illustrated, with a good selection of relevant absorption and circular dichroism spectra, and contains useful appendices of technical terms and of the unit systems in current use. It is recommended to all research workers concerned with chiroptical spectroscopy,

S.F. Mason is Professor in the Department of Chemistry, King's College, University of London, UK. as well as for general library reference.

\section{Insight into disorder}

\section{Praveen Chaudhari}

Models of Disorder. By J.M. Ziman. Pp.525. (Cambridge University Press: Cambridge, UK, and New York, 1979.) Hardback £25; paperback £12.50.

IN THIS book, Professor Ziman presents the first comprehensive and systematic review of the theoretical literature on the major forms of disorder. It is a remarkable book, both because of the subjects covered and because of the insight that the author brings to bear in explaining the generality and limitations of theoretical models.

The various forms of disorder are reviewed in the first three chapters. Chapter 1 is concerned with disorder where an underlying lattice can define the spatial arrangement of atoms. For example, compositional disorder in an alloy or spin disorder in an Ising model. This chapter contains a theme that is often repeated in the book - that of showing how the same mathematical model can be used to describe a number of situations which initially appear to be unrelated. In this instance the simple Ising model Hamiltonian is shown to apply to atomic interactions in a binary alloy. Its applicability to ferroelectric solids is briefly narrated. Chapter 2 deals with topological disorder characteristics of amorphous solids and liquids. Following a review of how the nature of disorder is described and modelled, there is a section on the analytical theories of the liquid state. In a natural progression, Chapter 3 contains a review of the statistical properties of random fields in continuous approximation. Chapter 4 has a brief and compact description of diffraction theory used in the study of disorder by experimental techniques. This is not a chapter that one can read and perform an experiment but rather one which contains the underlying physics and limitations on the information that can be extracted.

Chapter 5 has a detailed discussion of substitutional disorder. The success and limitations of the mean field approximations, the quasi-chemical approximation, the Bethe lattice and the pseudo-assembly method of Kikuchi are described. From these approximate solutions the author moves towards exact solutions of the one- and two-dimensional Ising model. The difficulties with obtaining a solution of the threedimensional Ising model are touched upon and graphical methods are introduced. The chapter concludes with a succinct review of scaling and renormalization developments of the last few years. Chapter 6 deals with the thermodynamics of topological disorder. As in the preceding chapter, Professor Ziman begins with onedimensional topological order and shows how genuine topological order cannot be 